\title{
'Re-appropriating" Facebook? \\ Web API mashups as Collective Cultural Practice
}

\author{
Stefan Werning
}

\begin{abstract}
In contemporary debates about socio-technical implications of software, the platform metaphor, the corresponding notions of architectures and ecosystems as well as the formatting of data to afford 'platformization' play a central role. This approach has certainly proven fruitful to assess the role of companies like Facebook in contemporary society. However, it characteristically overlooks the messiness of actual usage practices and those studies that do acknowledge the internal power struggles that subcutaneously shape platforms often take a top-down perspective, disregarding bottom-up processes of (re-) appropriation. To address this gap, the article outlines a method to study how users and semi-professional developers collectively frame the cultural imaginary of a platform by conducting a thoroughly comparative content analysis of mashups created using the Facebook Web API. The affordances of many individual mashups might be considered marginal; yet, the tool-assistant comparison allows for inferring common patterns of interpretation that characterize mashup creation as a mobile digital practice, which plays a key role in social media platform development.
\end{abstract}

\section{Introduction}

The article at hand investigates how users repurpose data and features of Facebook through mashups using the official API as well as the app-remixing service IFTTT, and how this collective practice re-situates their perceptions of Facebook use in everyday situations.

APIs are the interfaces that provide users structured access to big data sets ${ }^{1}$; apart from commercial APIs (Twitter, YouTube, Instagram etc.), which only offer part of their functionality for free, numerous public APIs exist as well. Most APIs require the user to sign up for their own developer key and in exchange provide (limited) access to a platform's methods and data. For instance, the "common

1 For a more comprehensive definition cf. e.g. https://www.programmableweb.com/ api-university/what-are-apis-and-how-do-they-work. 
scenarios" section on the Facebook API website ${ }^{2}$ lists publishing status updates, determining the friendship status between users, and scheduling Facebook Page posts as potential activities that can be implemented via a few lines of code using the official API.

As a topic in software studies, APIs are particularly relevant because recent examples not only facilitate implementing technical tasks (such as Facebook posts and search queries) but also legal and administrative functions, thereby blackboxing the underlying processes. For instance, the Betable API affords incorporating "real-money gambling" into any app or game, including the technical functionality (e.g. moving funds between Betable accounts) but, more importantly, also providing an 'interface' to the legal framework that offering a gambling service requires.

APIs have been tentatively discussed in terms of the political economy of social media platforms or, more specifically, as catalysts of "platformization" (Helmond 2015, 5). Tarleton Gillespie (2017) recently reassessed the platform as a metaphor that strategically frames both corporate and mainstream perceptions of social media technologies and businesses, arguing that the term hides both the hierarchies and corresponding power struggles that are usually invisible to regular users and the fact that these users themselves do not constitute one coherent community but multiple, sometimes contentious groups. This political economy of APIs is relevant for the argument below since APIs can change their modes of governance at any time; for example, in 2016 Instagram notably altered the modalities of its API integration with IFTTT and other aggregation services $^{3}$. Yet, while these complex and dynamic negotiation between platform providers, users, brands and not least mashup developers go beyond the scope of this article, the comparative analysis of mashups - each of which might seem hardly relevant in itself - can offer a valuable conceptual frames and material for follow-up research on the politics of API ecosystems. Thus, rather than conceptualizing platforms primarily from the perspective of data or rhetoric, it aims to rethink them in terms of practices or, more specifically, to analyse the products of API remixing and make systematic inferences about common rationales and approaches that inform this practice.

The professional development of mobile applications, which also includes platforms like Facebook, has already been the object of previous research, e.g. via semi-structured interviews with developers to investigate the role user participation plays during design and development (Mosemghvdlishvili and Jansz 2013). In contrast, this study foregrounds processes of bottom-up development in the form of (usually non-commercial) API remixing conducted by users with comparatively little technical knowledge. For that purpose, a comparative content analysis

2 Cf. https://developers.facebook.com/docs/graph-api/common-scenarios.

3 Cf. e.g. https://ifttt.com/blog/2016/05/a-change-in-how-instagram-works-with-ifttt. 
(Rössler 2012) of IFTTT applets ${ }^{4}$ as well as API remixes ${ }^{5}$ will be conducted to determine how users with lower and higher affinity for "computational thinking" (García-Peñalvo 2016) respectively re-envision Facebook use in conjunction with other services. The Facebook API was chosen as a case study because the service affords a broad range of possible uses and repurposing, and motivations for using the platform itself can differ from those of using individual functionalities (Smock et al. 2011). In total, the corpus consists of 490 IFTTT applets and 378 mash-ups on ProgrammableWeb using the Facebook API, which were scraped using Outwit Hub including basic metadata. Applets are referenced by unique numerical indices - for instance, [206] refers to an applet to automatically "wish Stephen Hawking a happy birthday on Facebook" by displaying a congratulatory message in the user's Facebook status on January 8 - and mashups by their names as listed on ProgrammableWeb, both in square brackets. The corresponding information, which also contains the verbatim quotes referenced below, can be found in an online Google Spreadsheet at http://bit.ly/2r8MEXO. In both cases, the data had to be manually cleaned up e. g. by flagging text from dynamically inserted ads since IFTTT applets are free and frictionless to create and the format has e. g. been abused for automated product placement.

\section{Approach}

Both IFTTT applets and API remixes can be summarized using the term mashups, which has been defined and institutionalized in the mid-20oos. For instance, Merrill (2006) argues that "one of the big catalysts for the advent of mashups was Google's introduction of its Google Maps API" and indeed mapping is one of the more prominent 'themes' in the corpus of API remixes below. Floyd et al. (2007) define (web) mashups as forms of user-driven innovation, which becomes especially visible in the IFTTT applets. These are small conditional statements that were formerly referenced as "recipes" ${ }^{\text {" }}$ and combine two supported services or devices; this cooking-related metaphor arguably emphasizes qualities like intuition and direct manipulatability, which stand in stark contrast to traditional software development..

Both types of mashups are essentially characterized by principles of bricolage, i.e. "making do" with (re)using and repurposing pre-existing material

4 A Google search for "facebook site:https://ifttt.com/applets/" yields almost 500 results, which have been scraped via Outwit Hub.

5 Programmableweb lists more than 370 mashups for the Facebook API, which have also been scraped via Outwit Hub; cf. https://www.programmableweb.com/category/all/ mashups?apis $=62918$.

6 Cf. e.g. https://www.wired.com/2013/12/with-location-ifttt-links-apps-to-your-realworld-activity/. 
(Vallgårda and Fernaeus 2015: 176). Yet, while traditional bricolage practices require "sampl[ing] media work" (Manovich 2013: 122), i.e. a conscious effort to 'appropriate' the material, mashups utilize ready-made components provided either by the APIs themselves or even an intermediary software like IFTTT or Zapier. Thus, these 'building blocks' (as well as the companies providing them) claim a much higher degree of agency than the materials in traditional bricolage contexts. This type of control can be understood with Galloway and Thacker (2004) as protocological, as APIs provide technical schemata for using features of a digital platform, which over time can contribute to the formation of cognitive schemata. Accordingly, multiple "protocols at play" during any technologically mediated situation such as a telephone call, "some [...] technical, some social" (14). These include the phone number as a technical identifier but also, in case of mobile phones, the social convention to ask where the person called currently is and whether they are free to speak. Similarly, web APIs as 'protocols' exercise indirect control by only exposing certain parameters ${ }^{7}$ but also, through repeated use, produce social norms of use. Moreover, they do not prescribe what users should do with them but making certain use cases easier and more appealing. From that angle, they can and will also be understood as conglomerates of 'game rules' that formulate an 'invitation to play' along, as play is characterized by a similar dialectic between imposing order and affording freedom (Huizinga 1949: $7 / 10)$.

\section{Academic contextualization}

APIs in general and the Facebook API in particular (Hogan 2009) have been used as research 'tools' in the digital humanities to investigate. For instance, Berry et al. (2015) use the Amazon API to conduct a tentative network analysis of the Digital Humanities discourse.

However, a close reading of one specific API and its impact on the social and technological imaginary of the corresponding platform is still missing in critical scholarship. Bodle (2011) takes a step in this direction by investigating how Open APIs facilitate interoperability and "regimes of sharing" between the major social media platforms (and Facebook in particular). He analyses Facebook's main Open API releases between 2006 and 2011 to determine how they constrain and reframe sharing on the platform. Complementary to this affordance-oriented perspective, this article looks at what users actually do with the API by comparing noncommercial mashups that use it.

7 For instance, the 'photo' construct in the Facebook API provides a list of predefined parameters such as width, height, the time of the last update and the location associated with the image; cf. https://developers.facebook.com/docs/graph-api/reference/ photo/. 
Most conspicuously, though, previous studies have attempted to 'map' the API ecosystem via network analyses (Evans and Basole 2016; Yu and Woodard 2009). The same approach, built on scraping all available connections around one specific platform, has also being applied to IFTTT (Ur et al. 2016). However, these mappings are static snapshots of a highly dynamic phenomenon, with constantly changing alliances. Moreover, they only focus on basic patterns in the ecosystem (e.g. the frequency of deliberate or unwitting imitation) rather than actual usage practices, i.e. while they identify relations between APIs they do not further elaborate on the qualities of these relationships. To complement this body of research, the content analysis conducted below does not rely on digital methods but focuses on identifying patterns in the functionality of API mashups as well as the rhetoric employed to describe them.

\section{Remixing web APIs as mobile digital practice and cultural technique}

The use of web APIs as analysed below constitutes a mobile digital practice because it organizes the mobility of data between different platforms. This definition requires a broader notion of mobility as proposed e.g. by Jensen (2013), who argues that mobility had been unjustly framed as a 'novel' paradigm in the context of the so-called "mobile media" (27). Instead, Jensen emphasizes the mobility of information, arguing that "for centuries, print media have disseminated information and entertainment within and between countries and across continents" (27).

From that angle, while previously data often constituted a value through controlled scarcity, web APIs constitute a new paradigm in which the mobility of data creates value. For instance, in his analysis of how TV companies can use social media APIs, Lahey (2016) defines the points of access between service that APIs define as a "sharing' ecology" (431), which helps users (in his case fans) move across platforms without actively switching between apps or web sites. He furthermore points out that "web APIs philosophically are not new" but that "contemporary interest in them is built on a type of structured openness and sharing, where many businesses want to give away access to their data and services but only on their terms" (433). That is, the code a company implements in its API functions and the data that pass through them become valuable primarily through 'movement'.

In line with the theme of this issue, the article at hand thus considers API remixing as a mobile digital practice and, more specifically, as a contemporary cultural technique. Practice-minded books on the subject already intuitively frame API use as "remixing” (Yee 2008). Moreover, as Manovich (2013: 167) notes, remixing is an iterative, self-sustaining practice that constantly forms new combinations, and "parts of these combinations enter into new remixes, ad infinitum". 
This self-sustaining quality can be observed in the API remixes but especially the IFTTT applets, e.g. as different variations on popular 'themes' such as automated birthday greetings reappear again and again in slightly different forms.

As will be elaborated below, API remixing should be conceptualized as an emergent cultural technique (Winthrop-Young 2013) because it constitutes a form of self-expression and of interpreting the world. While not yet feasible at scale, tools like IFTTT increasingly makes this practice wide-spread enough to be culturally formative, similar e.g. to how Adobe Flash afforded digital game making as a form of "social comment" (Thompson 2002) in the early 2000s. The concept of cultural techniques originated in post-war German media theory but has been tentatively introduced into Anglo-American media and culture studies discourse as well (Siegert 2013). According to Krämer and Bredekamp (2013), the conceptual relevance of cultural techniques has been to challenge the notion of culture as text and the primacy of "textual analysis and hermeneutics [as] the favorite model for the understanding of cultural orders" (21). Similarly, this article seeks to avoid reducing the cultural dynamics of API use to "monolithic immobility congealed in works" and, through its decidedly comparative approach, considers the individual mashups less as self-contained 'texts' than as manifestations of API remixing as a practice. As such, the patterns inferred from this material can serve as frames of reference for further praxeographic or netnographic studies that focus more on individual users or user communities.

Bogost (2009) addresses a similar ontological distinction regarding digital games, framing them (according to Jesper Juul) as lived experiences, which are shaped by the game as a rule system and can be studied via (digital) artefacts such as gameplay videos created in the process. This article attempts to adapt this ontological perspective to APIs as experienced 'in practice', pursuing the hypothesis that API remixing helps define the contours of Facebook's platform identity by keeping it 'mobile', in flux, through the constant use and repurposing of its core functionality.

More specifically, cultural techniques "also comprise sign systems such as musical notation or arithmetical formulas located outside the domain of the hegemony of alphabetical literacy" (20). In the case at hand, the API with its contingent selection of methods to access and manipulate data from an otherwise proprietary platform like Facebook prototypically fits that category. Like a new system of musical notation, e.g. graphical notation schemes introduced in the second half of the 2oth century (Evarts 1968), it becomes a gradually naturalized framework for self-expression and, even more importantly, its limitations and constraints appear increasingly 'natural' as well. Musical works that defy traditional notation like John Cage's 4'33" or John Zorn's Game Pieces make this naturalization visible by shaking up the corresponding conventions. Most of the API mashups analysed below suggest that the creators are still familiarizing themselves with these conventions but some uncommon combinations of APIs already suggest the potential to playfully subvert them. For instance, an IFTTT applet dating back 
to July 1oth 2014 [476] creates a "a digital 'Dead Man's Switch" by repurposing Google's Inactive Account Manager, sending out an automated tweet to officially 'proclaim' the user's death.

Below, the specificity of IFTTT mashup creation as opposed to the use of the official Facebook API are discussed separately, each with a focus on three characteristic patterns that manifested themselves during the comparison.

\section{Findings}

\section{IFTTT Applets}

The focus of this analysis lies on establishing and 'validating' a method to critically assess cultural implications of APIs, which would also be applicable similar tools like Zapier or Yahoo Pipes. Thus, it necessarily pursues an exploratory approach and does not claim the generalizability or statistical validity of instead findings. Instead, the comparative content analysis seeks to identify recurring patterns that characterize API remixing as a cultural technique by exhibiting distinct values and routines.

Werning (2016) points out that mobile applications, both those built into mobile operating systems and third-party apps, often require an inherently diachronic perspective because their affordances characteristically change constantly and often radically. This particularly applies to the APIs they offer, as methods and policies of data access often change over time or even become obsolete when the

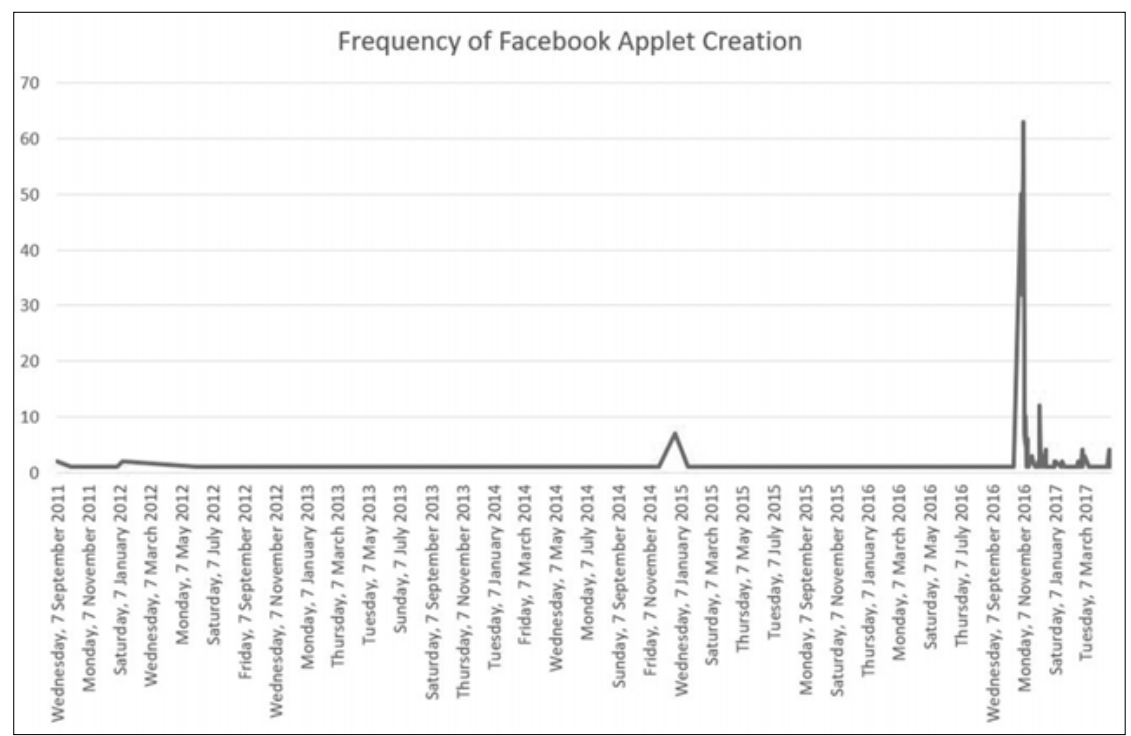

Figure 1: Facebook-themed applets created between 2011 and 2017. 
ecosystem around them changes ${ }^{8}$. This analysis does not pursue a decidedly diachronic approach, as the sample size would be too small to meaningfully investigate patterns of change over time, but both this section on applets and the following on mashups begin by contextualizing the corpus, pointing out fundamental changes that already become apparent during a cursory investigation.

The earliest applets that use the Facebook API, created between 2011 and 2014, focus a lot on the interoperability of Facebook and Google+, e.g. by enabling crossposting or posting via email as in applets [488], [486] and [485]. This suggests that during that time, Google+ was still interpreted as the most promising future competitor and alternative to Facebook. Moreover, the distribution of applets submitted over time shows that the rebranding from 'recipes' to 'applets' as well as the general overhaul of the IFTTT platform on November 2, 2016 significantly boosted user activity for a limited amount of time. While before, merely $1-2$ recipes using Facebook were created per month, the number shot up to 48 on November 2 , remained constant for a bit more than a week and dropped again to about three times the previous volume in mid-November.

\section{Self-automation}

As they constitute the main value proposition of IFTTT, automation practices constitute the bulk of user-created applets. From a media industries standpoint, critical positions on automation primarily focus on top-down production (Napoli 2014), e.g. the algorithmic prediction of demand or even creation of content. When, Lev Manovich referred to automation as a principle of new media (Manovich 2001: 32), he was still referring to algorithmic manipulation of digital content, e.g. a Photoshop filter changing colour or transparency values of all pixels in a digital image according to predefined rules. Through tools like IFTTT, human users can now apply similar principles to their own (digital) media use, creating 'macros' for their daily habits like recording actions in Photoshop "for tasks you perform frequently".

In the early 1980 s, the rapid advancement of robotics and microelectronics still prompted critical push-back against the cultural logic of automation. For instance, Sheridan, Vámos, and Aida (1983) posited that "technology should be individually designed to each culture" to ensure a proper match between the user's (mostly a worker's) physiology but also their "psychological and cultural characteristics" (605). In contrast, platforms like IFTTT celebrate automation through their curated collections of applets ${ }^{10}$ and institutionalize its underlying logic of efficiency and emergent complexity as desirable values through repeated use.

8 Major APIs usually offer deprecation schedules to ensure that their own ecosystem can adapt to the changes; cf e.g. https://developers.google.com/adwords/api/docs/sun set-dates.

9 Cf. https://helpx.adobe.com/photoshop/using/creating-actions.html.

10 Cf. https://ifttt.com/collections. 
Many applets are rather mundane and appear to be products of users familiarizing themselves with algorithmic logic itself (cf. e.g. applet [108], which allows for cross-posting content on Instagram and in a Facebook group). However, some culturally significant automation practices also become apparent, most notably updating the user's Facebook status message to 'celebrate' birthdays of people in the user's contact list [97, 112, 120]. These applets take up and further expand on an already established use of Facebook in popular culture. For instance, Techcrunch argues that "Facebook is where people celebrate birthdays online"11, pointing to recently introduced features such as Birthday Videos, through which Facebook attempts to solidify its 'claim' on this constitutive cultural practice. The abundance of birthday-related applet signals that users consolidates this assumption and at the same time suggests that the users' collective imaginary of what Facebook can be is notably shaped by the set of affordances currently on offer. Anticipating automation practices like this can also 'lead to' similarly automated follow-up practices. For instance, applet [284] thanks all Facebook friends for sending birthday greetings without any manual input required. Thus, while many users may experience social pressure due to Facebook's automated birthday reminders, applets enable them to use automation to 'relieve' themselves of that pressure, thereby leading to a closed loop of purely algorithmic exchange of birthday greetings on the platform. The same applies to other forms of social interaction that are less clearly tied to Facebook such as New Year's greetings [422].

These automation attempts occasionally eliminate established practices that had become part of Facebook 'culture', i.e. shared experiences among users of the platform that sometimes become visible because of the 'friction' they incur. For instance, several applets facilitate collecting all Facebook photos, in which the user has been tagged (cf. e.g. applets [417] or [429]). The creator of applet [417] demonstrates the habitualization of looking up references to oneself in the profiles of one's peers by claiming that his applet requires "no more scrolling through friends' feeds and albums to find those great pics". A study by Nicolai et al. (2009) indicates that narcissistic forms of web search such as self-googling have become deeply ingrained in many users' online experience. Yet, with the increasing pervasiveness of tools like IFTTT, the originally cumbersome processes, which required users to develop their own routines and 'shortcuts' and, thereby, became even more meaningful over time, are more and more discarded or, rather, 'offloaded' to an applet.

In more complex cases, automation manifests itself in the form of a 'communication with oneself', i.e. users explicitly set up algorithmic cues to call themselves to action or reaffirm their own values. For instance, applet [430] enables users to 'manage' their music fandom by uploading the cover art of newly favourited music albums on Deezer to a dedicated photo album on Facebook. In doing so, they arguably create a socio-technical system for themselves and the technolo-

11 Cf. https://techcrunch.com/2016/07/28/facebook-birthday-videos/. 
gies (i. e. web platforms) they use. Niederer and van Dijck (2010) use that term e.g. to describe how bots on Wikipedia are designed to produce content that is particularly aimed at strengthening the community (1378), i.e. at spurring other users into action. For example, Rambot "pulls content from public databases and feeds it into Wikipedia", encouraging (human) users to flesh out the resulting incomplete articles; subsequently, many "bot-generated articles on American cities and counties [are] corrected and complemented by human editors" (1378). The Deezer applet above suggests that individual users employ applets in a similar way to create their own personalized socio-technical systems. The same applies e.g. in the case of applet [372], which emails the user once they have been invited to an event through Facebook, thus creating partially redundant content as 'breadcrumbs' for oneself to follow.

Finally, users employ automation practices also to historicize their own Facebook activity. For instance, applet [384] creates an automatic backlog of all status messages in a Google spreadsheet, thereby creating a focused form of 'diary' that would not be feasible to maintain by other means. Applets like this reframe Facebook as a tool to track one's own emotional development over time independent from factual or even quantified data, as no contextual information is saved alongside the status messages.

\section{'Brand' management}

A second recurring pattern is the use of applet for 'self-branding' purposes, i.e. to present oneself according to brand logic. First, this involves maintaining a coherent impression across social platforms. For example, applet [111] automatically synchronizes the user's profile pictures on Twitter and Facebook.

Second, several applets automatically inform 'followers' of newly created content, e.g. if the user starts a new Twitch stream [113], is currently live-vlogging via Periscope [442] or has recently uploaded music on SoundCloud [427]. Therefore, applets help user cope with the pressure of having to produce new content on a regular basis; e. g. applet [126] allows for populating one's Facebook timeline with blog content via RSS feeds. It is important to note that applets like this re-appropriate the timeline as a key affordance of Facebook, turning it from a backlog of events in the user's life ${ }^{12}$ to a 'news ticker'. Another technical aspect of Facebook that users characteristically re-appropriate via applets is the status message, which has traditionally been used as flexibly as possible e.g. to create "a feeling of connectedness between users" (Köbler et al. 2010) through the topicality of its content. However, algorithmic manipulation takes that flexibility to

12 Shortly after its introduction, the feature was summarized as "show[ing] the story of your life, as you choose to tell it or as Facebook has recorded it”; cf. http://uk.pcmag. com/internet-products/66981/feature/12-things-you-should-know-about-facebooktimeline. 
unprecedented levels. For instance, applet [343] updates the status with every song a user listens to on Echo. Thus, while the status message used to be an asynchronous and quintessentially personal self-assessment of the user's current situation, the aforementioned applets turn it into an affordance that contributes to the specific "realtimeness" (Weltevrede, Helmond, and Gerlitz 2014: 130) of Facebook as a platform.

Third, applets allow for rationalizing content posting by using Facebook in tandem with a scheduling application to plan one's social media activity. According to brand logic, content should be 'spread out' evenly over time, as this maximizes the recognizability of brand identity and enhances user 'loyalty' by making it easier for them to integrate brand messages into their daily routines (cf. e.g. Broussard 2000). While previously only specialized applications like Buffer ${ }^{13}$ used to offer that functionality, IFTTT applets make it accessible to anyone. This logic is intuitively applied to individual self-presentation on social media platforms as well. Applications of corporate 'rhetoric' to aspects of private life ${ }^{14}$ have already been promoting neoliberal subjectivity (McGuigan 2014) for decades. Yet, applets like these are more influential than language use, allowing users to 'performing' neoliberal rationales online through repeated practice, which makes them 'feel' all the more 'natural' over time.

Finally, the creation and use of IFTTT applets gradually blurs the boundary between private and professional applications. For example, applet [100] targets semi-professional users by implementing "automated Ads reporting into shared Google Drive for marketing teams”. More importantly, private and professional entities appear in the same format on the service. For instance, applet [98] "automatically post [s] a celebratory status on Facebook" if the user reaches a set step goal on their pedometer. While this appears like a personal attempt at self-optimizing, the applet was published by the consumer electronics company Withings rather than an individual user to promote their wearable fitness trackers. As applets on IFTTT are curated by the platform according to topic area, this dispositif suggests a kind of ongoing 'conversation' between companies and individual users. Yet, it also requires to be aware of who is 'speaking', as companies use the same format to foster use of their services ${ }^{15}$.

13 Cf. https://buffer.com/.

14 A 2014 article from De Groene Amsterdammer (cf. https://www.groene.nl/artikel/ikis-een-start-up) aptly summarized the debate by proposing that Foucault's original claim - that people gradually reinvent the "self as enterprise" - may become all the more urgent due to the rise of startups to the status of a social 'role model'.

15 E.g. applet [404] is an applet created by Google that uses its voice recognition capabilities to let users post by 'talking' to Facebook. 


\section{Filling 'gaps' in affordance networks}

Finally, several applets indicate that users become more and more adept at understanding affordance networks ${ }^{16}$, i.e. how functionalities between related services overlap, but also which expectable functionalities are 'missing'. For instance, the creator of applet [477] claims that the applet "fixes" the "lack of notifications" on Tinfoil, which they recently switched to because of privacy concerns. Thus, applets are used as workarounds to 'remedy' these omissions. Similarly, applet [470] compensates for the inability of the Xbox One to share screenshots via Facebook by posting them first to Twitter and then copying them automatically to Facebook. This applet illustrates a common principle that users intuitively 'learn' by using applets, namely the need to use a specific nomenclature (in this case for naming the Xbox screenshots posted to Twitter) so that they can be reliably detected and manipulated using simple algorithmic means such as the eponymous if-this-then-that logic. For instance, applets [457], [385] and [446] all illustrate the principle using different examples. On a related note, the use of applets 'teaches' users about the kind of structured data that Facebook provides. For instance, applet [413] makes Facebook notifications available on services that don't usually support them by tapping into the personalized RSS feed that Facebook generates for all users' notifications, but which is not part of the regular interface and, thus, 'invisible' to many users.

\section{Facebook API remixes}

Compared to IFTTT applets, remixes using the official Facebook API are usually much less focused on one platform but rather combine multiple APIs to reach more freely defined goals. Thus, while applets are often conceived based on technological feasibility, API remixes represent visions of social media use in which any individual API is usually but one building block. Moreover, since API remixes require more active maintenance to develop a stable user base, they are much more volatile than applets. Many mashups in the corpus, still listed on ProgrammableWeb, are already discontinued and the rhetoric employed in some descriptions suggests that longevity is considered a value in itself. For instance, [Noozly] simply claims to provide "social news since 2009 ", mimicking the same rhetoric that many regional brick-and-mortar businesses have traditionally used to evoke trust and authority. Therefore, considering basic metadata characteristics is a useful step to provide basic orientation for a more interpretive approach.

Upon parsing the API descriptions through Voyant Tools ${ }^{17}$, it becomes evident that the most popular terms like 'social' (52) are expectedly rather generic. Similarly, the term 'friends' (43) is used only slightly more often than 'users' (41). The

16 Cf. e.g. http://www.normanjackson.co.uk/creativehe/category/affordance.

17 Cf. http://beta.voyant-tools.org/. 
most relevant related activities are search (38), photos (21), maps (16) and music (15), i.e. mashups on audiovisual content are particularly appealing. Consequently, the platforms mentioned most prominently in conjunction with Facebook are Google (27), Twitter (21), YouTube (15), Flickr (10) and Foursquare (5). Programmableweb lists 30 API mashups that were already created in 2007 , only one year after Facebook became publicly available. A few of these mashups anticipate use cases that would only later become billion-dollar industries.

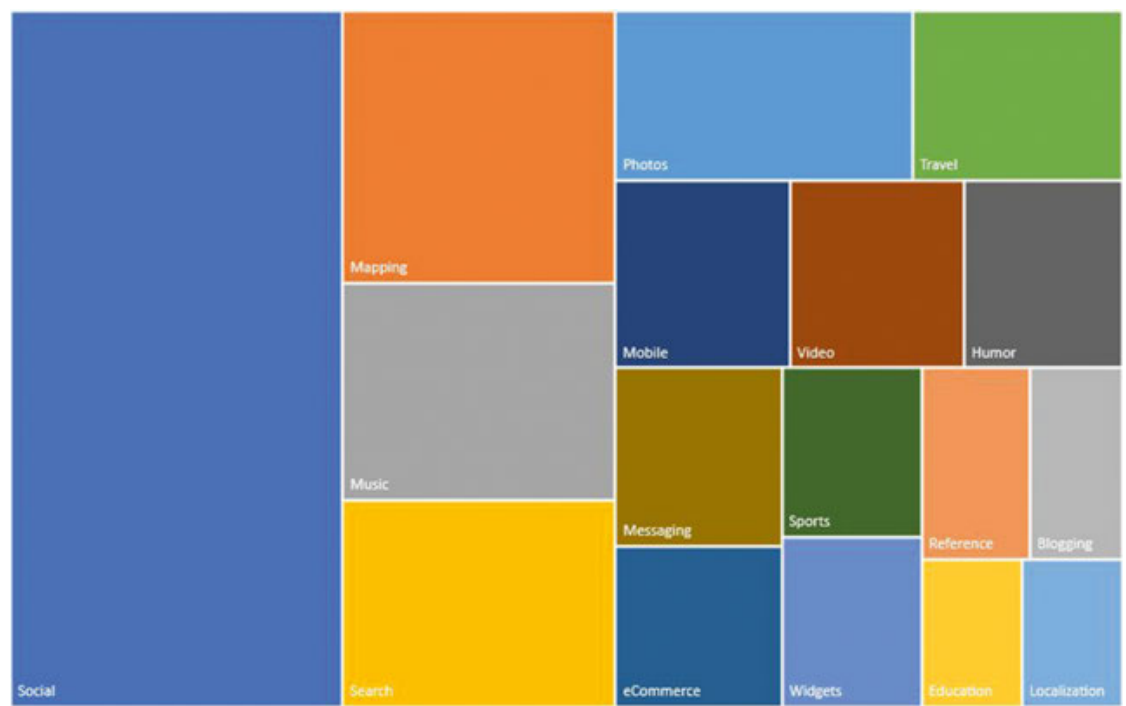

Figure 2: Most popular genres of Facebook API mashups.

Number of Facebook API mashups

(via programmableweb.com)

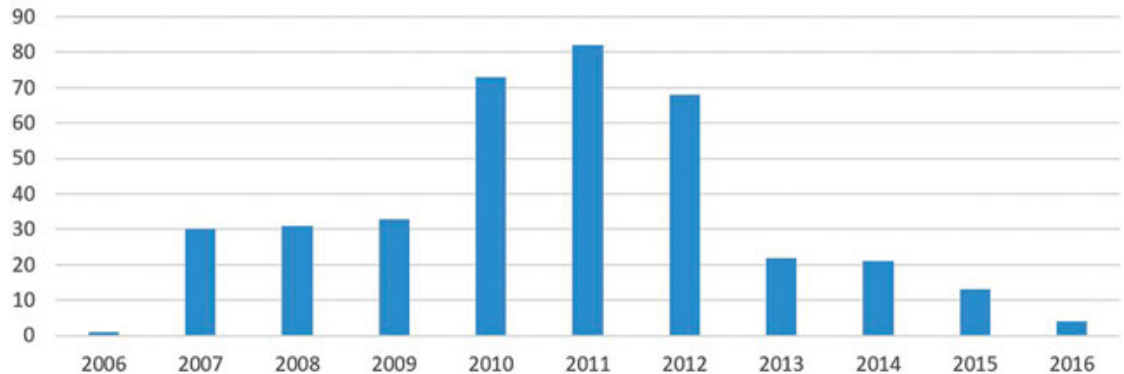

Figure 3: Development of Facebook API mashups between 2006 and 2016.

For instance, [Rendezbook] - the earliest listed mashup from November 2006 is summarized as "a crush system for Facebook" by enabling users to "tell the system who you would like to become better friends with". While services like OkCupid (2004) and Match.com (1993) had already been established online dating 
platforms, the format only later, in the early 2010s, became part of mainstream social media use through Tinder (2012) and concurrent offerings. Remixing of the Facebook API appears to have peaked in 2011 with a little more than 80 mashups registered during that year.

While later mashups combine multiple APIs as needed, early examples demonstrate the sometimes-frantic rush to carve out a niche in the Facebook 'economic ecosystem ${ }^{18}$. For instance, [Mosoto] (Feb. 2007) positioned itself as a new layer "on top of your Facebook account"19 that characteristically positions filesharing as a quasi-social practice, by integrating it directly into the chat interface. [Facebook Friend Plotter] (Apr. 2007) visualized the location of Facebook friends in the UK on a map screen, thereby providing an alternative view on the user's expanding circle of friends. [Facebook Friends Map] (2009) later applied that principle on a global scale. This came at a time when news media began pointing out that Facebook users were forming friend relationships "as if they were collecting action figures, stamps or collectables"20. Finally, [Zuckerbucks] (Jul. 2007) introduced a points system that could be "use[d] like play money on Facebook", years before the platform would officially introduce its own (already discontinued) currency Facebook Credits in 2011.

Paul Ricoeur famously referred to literature as "a vast laboratory in which we experiment with estimations, evaluations, and judgments of approval and condemnation", which consequently requires thinking of narrativity as "a propaedeutic to ethics" (Ricoeur 1992: 115). Thus, through literature a society might "prototype' potential future constellation, which may not yet be feasible at the respective time of writing, and simultaneously constructs a basis for moral judgment. The multiplicity of mashups that (re)interpret the social vision built into Facebook's functionality, especially through redundancies and slightly varying takes on similar themes, arguably performs a similar function. Fluck (1997) makes a similar claim as Ricoeur, arguing that American literature has been producing cultural imaginaries that in some cases later became social reality or paved the way for social changes; by analogy, the process of creating mashups allows for exploring social uses and potential futures of Facebook as a platform and the community that emerged around it. For instance, [Two Degrees] (2007) allowed for searching through the Facebook friend lists of friends to "find people who should be on your own list", long before algorithmic recommendation of potential

18 The term is used following Rachel Rosmarin's definition, who used it to analyze how gaps in the MySpace functionality offered numerous opportunities for small supplementary businesses to fill, which could not exist without the platform's large user base to sustain them; cf. https://www.forbes.com/2006/04/07/myspace-googlemurdoch-cx_rr_0410myspace.html.

19 Cf. https://techcrunch.com/2007/02/07/mosoto-share-files-and-chat-on-facebook/.

20 http://www.telegraph.co.uk/news/science/science-news/3306173/Facebook-studyreveals-users-trophy-friends.html. 
contacts $^{21}$ institutionalized the idea of 'importing' social relations like one would copy and paste digital data from one list into another. Platforms like Facebook are constantly in flux, as their underlying algorithms are incessantly tweaked (Bogost 2016) and interface elements added, removed and changed. Therefore, their "technological imaginary" (Lister et al. 2009: 66/67), i.e. the consensual notions of how they will evolve and which role they should play in society, is characteristically blurry. This spectrum of potential interpretations - both on behalf of the users and the designers - is also summarized as the "interpretative flexibility" (Bijker et al. 2012: 34) of technological artefacts in a broader sense. The creation and use of API mashups thus makes this flexibility visible by expressing possible design futures of Facebook in the form of discrete, comparable digital objects.

\section{Invitation to play}

The corpus of remixes also illustrates that web API use is not purely guided by utilitarian rationales but e.g. also perceived as "fun" 22 or as an invitation to "play"23. For instance, the popular online platform Any API offers test consoles for about 270 APIs that affords playfully combining data and methods by making the results instantly visible. According to its self-description, Any API suggests the cyclical principle "Explore - Discover - Try - Build" as the ideal way to use APIs ${ }^{24}$, which also essentially describes the way a player approaches a game. Previous research conducted within the digital media industry, e.g. on the emergence of playful GUIs in the mid-199os (Yager et al. 1997) or on playful uses of communication technology in the household (Lindley, Harper, and Sellen 2010), shows that playful use can indeed be tapped into as conduit for innovation. While these studies use the notion of play rather intuitively, API remixing exhibits several properties that e. g. Huizinga (1949) considers characteristic of play. It is a "voluntary activity" (7) rather than a task conducted for an external purpose; accordingly, the vast majority of mashups considered in this article have not been created in a commercial context. Furthermore, play is "not 'ordinary' or 'real' life" (8), which is to say it is detached from real-world consequences and allows for practicing specific skills in a safe space. Similarly, mashup creation- especially compared to traditional software development - characteristically affords 'trial and error' because it usually requires no significant investment of time or money and

21 For instance, a post on the LinkedIn blog from 2015 argues that all major social networks rely on "their trademark Friend suggestion algorithms"; cf. https://www. linkedin.com/pulse/how-does-linkedin-gives-you-people-may-know-suggestionsatiq.

22 Cf. e.g. https://www.quora.com/What-are-some-cool-fun-APIs.

23 Cf. e.g. https://www.reddit.com/r/webdev/comments/3wrswc/what_are_some_fun_ apis_to_play_with/.

24 Cf. https://www.any-api.com/. 
because it emphasizes playful bricolage, i.e. 'making do' with ready-made functions created by the API provider, instead of pre-emptive planning. However, while Huizinga argues that technology fosters "commercial competition" and thus arguably diminishes the "immemorial sacred play-forms" (200), API mashups demonstrate that particularly digital technologies not only streamline processes but also create new niches for playfulness (Sicart 2014).

Apart from finding uncommon combinations of APIs, one distinct pattern of play is the application of the 'rules of play' of online social networks to different audiences such as vegan users [VeganHunter], business travelers [Arrivedin. com] or "orchid lovers" [Orcheeder]. Moreover, remixes playfully re-enacting the nomenclature of contemporary mobile applications. These include nonsensical neologisms and misspelling (e.g. [Kiwifruut], a social YouTube discovery service), common morphemes (e.g. [Flixster], imitating iconic names like Napster or Friendster), active verbs phrased like an appeal (e.g. [ConnectMyRide] or [Stay in Touch]), and vowel omission (as in [NetTickr] or [Listnr]).

\section{Promoting purchasing as quasi-social activity}

While the rapid growth of Groupon turned social shopping into a dedicated (mainstream) media practice, [fflap] (2010) is but one of many API mashups that contributed to bridge the epistemic gap between ecommerce and online social interaction by combining eBay and Facebook functionality through their respective APIs. [MyShopping Facebook] (2007) enables users to "to connect with friends, co-workers, or family on your daily shopping activities"; similarly [Mallicious Social Shopping] (2007) "leverages real life relationships on Facebook". Both early mashups reframe shopping as a complementary form of online social interaction by combining the then-newly available Facebook API with ecommerce APIs. Other mashups helped discursively stabilize this framing. For instance, [50 Shops] (2011) claimed to enable "buyers to tap into the wisdom of crowds to help with their buying decisions"; that is, it referenced Pierre Levy's dictum, which at that time had trickled down into popular discourse, to make collaborative buying on Facebook appear both topical and natural (i.e. 'wise'). Over time, the aforementioned fragmentation of audiences also occured in this mashup 'genre'. For instance, [SuittsMe] (2011) focuses specifically on clothes shopping while [Arms Dealer] (2011) encourages users to frequent and review gun shops and shooting ranges.

While many mashups are evidently designed to boost sales by fostering product-oriented discussions, a few more recent examples also suggest that using the Facebook API in conjunction with ecommerce APIs can also promote 'price literacy', thereby making users potentially more resilient against these very same commercialization attempts. For instance, [GrabEvery] (2012) facilitates the use of coupons and coupon codes by affording price comparison to a previously unprecedented extent; this use of digital technology contributes to an ongoing 'profes- 
sionalization' of purchasing practices that (Schwartz 2010) calls a subculture of "retail hacking". [PriceZombie] (2015) fulfills a similar purpose as a "price tracker for numerous retail stores and a comparison shopping site".

\section{Providing 'data types' for self-expression}

Self-expression on social media platforms is defined by its, more or less visible, constraints such as the 140-character-limit on Twitter but also, more recently, Facebook's decision to replace the simple like button with six predefined "Reactions" in early $2016^{25}$. These 'data types', i. e. structured formats for expressing complex information, directly frame the self-description of those using them. As Drucker and Haas (2017) argue in a broader, almost McLuhanesque sense, "the way in which information is structured is the real information" (119) [translation by the author of this article]. More specifically, (Anderson 1991) shows how the census with its grid-like structure to categorize families has been an actively used tool for colonial governance. Similarly, the six ideal-typical Reactions constrain the spectrum of potential emotional states and thereby narrow down the user's selfdescription, which made them as well as related 'data types' potential objects of contention and some remixes attempt to expand that focus. For instance, [Expin. me] affords "mid to long format expression", i. e. short story-length user contributions, arguing that Facebook posts are not suitable for conveying more idiosyncratic ideas. Moreover, it supports social writing in "Hindi, Marathi, Kannada, Tamil, Bengali or Spanish”. [Expressi] (2011) combines providing new categories for self-expression with the aforementioned focus on social shopping. It asks users "which item is the most emotionally and personally relevant for [them]" by combining the Amazon Product Advertising API with sharing capabilities via Facebook. This rather unusual combination on the one hand substantiates the common perception that users are supposed to engage in "meaningful interaction with products" (de Medeiros 2014, 16) and that contemporary product design aims for an affective relationship with commodities. However, it also illustrates that these concepts are shaped by technological feasibility as the Amazon Product Advertising API provides ready-made access to products across all the major region-specific Amazon websites as well as discoverability functionality such as "Product Search, Customer Reviews, Similar Products, Accessories, [and] Wish Lists" ${ }^{\prime 26}$.

Finally, coming back to the initial notion of APIs organizing the mobility of data across platforms, many mashups institutionalize a cross-platform perspective. That is, because multi-API use makes it particularly easy to obtain and compare data from different services, it makes this inherently comparative perspective

25 Cf. e.g. https://www.wired.com/2016/02/facebook-reactions-totally-redesigned-likebutton/.

Cf. https://affiliate-program.amazon.com/gp/advertising/api/detail/main.html. 
feel particularly 'natural'. For instance, [Social page authority checker] (2014) aggregates and compares quantitative data for multiple websites from Facebook but also Google+, LinkedIn and Pinterest to calculate an overall averaged "social media authority" score. [Rápido] applies the same principle to posting information, enabling users to simultaneously post to Facebook, Twitter, Google+, and Foursquare. Through repeated use and incorporation into everyday media routines, these mashups foster a dialectic perspective. On the one hand, online social networks become increasingly fragmented, tailored to increasingly narrow audiences; for instance, Craig (2016) demonstrates the increasing fragmentation of online communities with regard to online dating platforms such as The League, Sparkology, the Dating Lounge, and Luxy. However, the previously mentioned examples at the same time cause the different networks to seemingly 'converge' within the daily practices and, thus, the perception of the users.

\section{Outlook}

The comparative content analysis shows that, with a few exceptions, the collective imaginary of what Facebook can or should be is still narrowly framed by technical feasibility (e.g. backups and cross-posting) as well as the kind of functionality that Facebook 'claims' for itself (e.g. birthday greetings). This is most likely since API mashup creation is still a marginalized practice with comparatively little social interaction between creators and users that would allow for 'refining' the remixes as forms of self-expression. This is relevant because rather than outwardly visible terms of service that would dictate the limits of app remixing, these forms of governance (Light, Burgess, and Duguay 2016: 10) are embedded in the practical affordance of the Facebook API (and IFTTT as an intermediary). Genuinely playful uses of remixes or even applets, e.g. applet [400] posting a steady stream of martial arts memes to the user's Facebook account, are still comparatively rare. While this is plausible in the case of remixes, which are often created by users with 'professional' ambitions, who want to become part of the industry and, thus, expectedly 'play by its rules', it is more surprising in the case of applets. Moreover, most users characteristically direct their mashups at an undefined, inherently global audience, with only few exceptions. For instance, applet [355] was created by a user from the Austrian state of Vorarlberg to monitor speed limit enforcement in his area and share related news with a group of Facebook friends.

The results of this exploratory study map out several highly relevant areas for follow-up research. As suggested above, a distinctly diachronic content analysis, potentially including IFTTT applets using all the major online social networks, could be a fruitful direction for follow-up research based on the approach outlined in this chapter. Moreover, as suggested above, applets and API remixes differ in terms of the technical hurdles they impose on the user as remixes require a higher level of both information literacy (Asselin et al. 2011) and algorithmic 
literacy. While this could not be systematically factored into the analysis above, the notion of literacy is another useful axis in further studies on API use. Finally, investigating different corpora of IFTTT applets can produce interesting results, particularly if not narrowed down by technology (like Facebook) but by practice. For instance, IFTTT itself curates selections of applets e. g. by season (Christmas) or theme (e.g. work-outs). APIs as catalysts of mobile digital practices are increasingly incorporated into curricula (Robillard and Deline 2011; Olsen/Moser 2013), which gradually bridges the gap between programmers and non-programmers and helps refine API mashup creation as a genuinely digital cultural technique.

\section{References}

Anderson, Benedict (1991): Imagined Communities: Reflections on the Origin and Spread of Nationalism, New York, NY: Verso.

Asselin, Marlene/Dobson, Teresa/Meyers, Eric M./Teixiera, Cristina/Ham, Linda (2011): "Learning from YouTube: An Analysis of Information Literacy in User Discourse." In: Proceedings of the 2011 iConference, Washington: ACM.

Berry, David M./Borra, Erik/Helmond, Anne/Plantin, Jean-Christophe/Rettberg, Jill Walker (2015): “The Data Sprint Approach: Exploring the Field of Digital Humanities through Amazon's Application Programming Interface." In: Digital Humanities Quarterly 9/3, article 222, (http://hdl.handle.net/11245/ 1.502979).

Bijker, Wiebe E./Hughes, Thomas P./Pinch, Trevor/Douglas, Deborah G. (eds.) (2012): The Social Construction of Technological Systems: New Directions in the Sociology and History of Technology, Cambridge, MA: MIT Press.

Bodle, Robert (2011): "Regimes of Sharing. Open APIs, Interoperability, and Facebook." In: Information, Communication \& Society 14/3, pp. 320-37.

Bogost, Ian (2009): “Videogames Are a Mess.” Keynote Speech at DiGRA. (http:// bogost.com/downloads/Videogames are a Mess slides.pdf).

Bogost, Ian (2016): “Go Tweak Yourself, Facebook.” In: The Atlantic, April 28, (http://www.theatlantic.com/technology/archive/2016/04/go-tweak-yourselffacebook $/ 480258 /)$.

Broussard, Gerard (2000): "How Advertising Frequency Can Work to Build Online Advertising Effectiveness.” In: International Journal of Market Research 42/4, pp. 439-57.

Craig, Elise (2016): "Niche Dating Apps Like the League Are Icky and Bad for Love." Wired Magazine, June 6, (https://www.wired.com/2016/o6/why-tinderis-bad/).

de Medeiros, Wellington Gomes (2014): "Meaningful Interaction with Products." In: Design Issues 30/3, pp. 16-28.

Drucker, Johanna/Haas, Annika (2017): “Digital Humanities als Epistemische Praxis.” In: Zeitschrift Für Medienwissenschaft 16, pp. 114-25. 
Evans, Peter C./Basole, Rahul C. (2016): "Revealing the API Ecosystem and Enterprise Strategy via Visual Analytics.” In: Communications of the ACM 59/2, pp. 26-28.

Evarts, John (1968): “The New Musical Notation: A Graphic Art?” In: Leonardo $1 / 4$, pp. 405-12.

Floyd, Ingbert/Jones, M. Cameron/Rathi, Dinesh/Twidale, Michael (2007): "Web Mash-Ups and Patchwork Prototyping: User-Driven Technological Innovation with Web 2.o and Open Source Software.” In: Proceedings of the 4oth Annual Hawaii International Conference on System Sciences, IEEE Computer Society.

Fluck, Winfried (1997): Das Kulturelle Imaginäre: Funktionsgeschichte Des Amerikanischen Romans, 1790-1900, Frankfurt/Main: Suhrkamp.

Gillespie, Tarleton (2017): “The Platform Metaphor, Revisited” In: HIIG Science Blog, (https://www.hiig.de/en/blog/the-platform-metaphor-revisited/).

Helmond, Anne (2015): “The Platformization of the Web: Making Web Data Platform Ready.” In: Social Media + Society 1/2, pp. 1-11.

Hogan, Bernie (2009): "A Comparison of On and Offline Networks through the Facebook API.” In: Social Science Research Network Working Paper Series, December 18, doi:10.2139/ssrn.1331029.

Huizinga, Johan (1949): Homo Ludens. A Study of the Play-Element in Culture, London, Boston and Henley: Routledge \& Kegan Paul.

Jensen, Klaus Bruhn (2013): “What's Mobile in Mobile Communication?" In: Mobile Media \& Communication 1/1, pp. 26-31.

Köbler, Felix/Vetter, Céline/Riedl, Christoph/Leimeister, Jan Marco/Krcmar, Helmut (2010): "Social Connectedness on Facebook - An Explorative Study on Status Message Usage.” In: Americas Conference on Information Systems, Lima, Peru. doi:10.2139/ssrn.1953431.

Krämer, Sybille/Bredekamp, Horst (2013): "Culture, Technology, Cultural Techniques - Moving Beyond Text.” In: Theory, Culture \& Society 30/6, pp. 20-29.

Lahey, Michael (2016): "Invisible Actors: Web Application Programming Interfaces, Television, and Social Media." In: Convergence: The International Journal of Research into New Media Technologies 22/4, pp. 426-39.

Light, Ben/Burgess, Jean/Duguay, Stefanie (2016): "The Walkthrough Method: An Approach to the Study of Apps." In: New Media and Society First published November11, (http://journals.sagepub.com/doi/abs/10.1177/1461444816675438). Lindley, Siân E./Harper, Richard/Sellen, Abigail (2010): “Designing a Technological Playground: A Field Study of the Emergence of Play in Household Messaging." In: CHI 2010: We Are Family, Atlanta, GA: ACM, pp. 2351-6o.

Lister, Martin/Dovey, Jon/Giddings, Seth/Grant, Iain/Kelly, Kieran (eds.) (2009): New Media: A Critical Introduction, Abingdon: Routledge.

Manovich, Lev (2001): The Language of New Media, Cambridge, MA: MIT Press. Manovich, Lev (2013): Software Takes Command, New York/London: Bloomsbury. McGuigan, Jim (2014): “The Neoliberal Self." In: Journal of Current Cultural Research 6/1, pp. 223-40. 
Merrill, Duane (2006): “Mashups: The New Breed of Web App.” In: IBM Web Architecture Technical Library, (http://www.citeulike.org/user/CEAG/article/ 7359315).

Mosemghvdlishvili, Lela/Jansz, Jeroen (2013): “Negotiability of Technology and Its Limitations: The Politics of App Development.” In: Information, Communication \& Society, $16 / 10$, pp. $1596-1618$.

Napoli, Philip M. (2014): “On Automation in Media Industries: Integrating Algorithmic Media Production into Media Industries Scholarship." In: Media Industries Journal, 1/1, (http://www.mediaindustriesjournal.org/index.php/mij/ article/view/14/60).

Nicolai, Thomas/Kirchhoff, Lars/Bruns, Axel/Wilson, Jason/Saunders, Barry (2009): "Google Yourself! Measuring the Performance of Personalized Information Resources.” In: First Monday 14/12, doi:10.5210/fm.v14112.2683.

Niederer, Sabine/van Dijck, José (2010): “Wisdom of the Crowd or Technicity of Content? Wikipedia as a Sociotechnical System.” In: New Media \& Society 12/8, pp. 1368-87.

Olsen, Timothy/Moser, Kathleen (2013): “Teaching Web APIs in Introductory and Programming Classes: Why and How." In: Proceedings of the SIG-ED: IAIM Conference. Association for Information Systems (AIS), (http://aisel.aisnet.org/ siged2013/16).

Ricoeur, Paul (1992): Oneself as Another, Chicago: University of Chicago Press.

Robillard, Martin P./Deline, Robert (2011): “A Field Study of API Learning Obstacles.” In: Empirical Software Engineering 16/6, pp. 703-32.

Schwartz, Matt (2010): “The Coupon Rebellion.” In: Wired Magazine 18/12, pp. $188-193 \& 224$.

Sheridan, Thomas B./Vámos, Tibor/Aida, Shuhei (1983): "Adapting Automation to Man, Culture and Society." In: Automatica 19/6, pp. 605-12.

Sicart, Miguel (2014): Play Matters, Cambridge, MA: The MIT Press.

Siegert, Bernard (2013): "Cultural Techniques: Or the End of the Intellectual Postwar Era in German Media Theory.” In: Theory, Cultur \& Society 30/6, pp. 48-65.

Smock, Andrew D./Ellison, Nicole B./Lampe, Cliff/Wohn, Donghee Yvette (2011): "Facebook as a Toolkit: A Uses and Gratification Approach to Unbundling Feature Use.” In: Computers in Human Behavior 27/6, pp. 2322-29.

Thompson, Clive (2002): "Dot-Columnist. Online Video Games Are the Newest Form of Social Comment.” In: Slate, August 29, (http://www.slate.com/articles/ technology/webhead/2002/o8/dotcolumnist.html).

Ur, Blasé/Ho, Melwyn Pak Yong/Brawner, Stephen/Lee, Jiyun/Mennicken, Sarah/ Picard, Noah/Schulze, Diane/Littman, Michael L. (2016): “Trigger-Action Programming in the Wild.” In: Proceedings of the 2016 CHI Conference on Human Factors in Computing Systems - CHI '16, New York: ACM Press, pp. 3227-31. 
Vallgårda, Anna/Fernaeus, Ylva (2015): “Interaction Design as a Bricolage Practice.” In: Proceedings of the Ninth International Conference on Tangible, Embedded, and Embodied Interaction - TEI '14, New York: ACM Press, pp. 173-80.

Weltevrede, Esther/Helmond, Anne/Gerlitz, Carolin (2014): "The Politics of RealTime: A Device Perspective on Social Media Platforms and Search Engines.” Theory, Culture \& Society 31/6, pp. 125-50.

Werning, Stefan (2016): “The Home Screen as an Anchor Point for Mobile Media Use - Technologies, Practices, Identities.” In: NECSUS European Journal of Media Studies Autumn/Special Issue \#Home, (http://necsus-ejms.org/thehome-screen-as-an-anchor-point-for-mobile-media-use-technologies-practi ces-identities/).

Yager, Susan E./Kappelman, Leon A./Maples, Glenn A./Prybutok, Victor R. (1997): “Microcomputer Playfulness: Stable or Dynamic Trait?" In: The DATA BASE for Advances in Information Systems 28, pp. 43-51.

Yee, Raymond (2008): Pro Web 2.0 Mashups: Remixing Data and Web Services, Berkeley, CA: Apress.

$\mathrm{Yu}$, Shuli/Woodard, C. Jason (2009): “Innovation in the Programmable Web: Characterizing the Mashup Ecosystem.” In: Service-Oriented Computing ICSOC 2008 Workshops, Berlin, Heidelberg: Springer, pp. 136-47. 